\title{
Comparative Study of Trajectory Tracking Control for Automated Vehicles via Model Predictive Control and Robust H-infinity State Feedback Control
}

\author{
Kai Yang ${ }^{1}$, Xiaolin Tang ${ }^{1^{*}}$ (D) Yechen Qin², Yanjun Huang ${ }^{3}$, Hong Wang $^{4}$ and Huayan $\mathrm{Pu}^{1}$
}

\begin{abstract}
A comparative study of model predictive control (MPC) schemes and robust $H_{\infty}$ state feedback control (RSC) method for trajectory tracking is proposed in this paper. The main objective of this paper is to compare MPC and RSC controllers' performance in tracking predefined trajectory under different scenarios. MPC controller is designed based on the simple longitudinal-yaw-lateral motions of a single-track vehicle with a linear tire, which is an approximation of the more realistic model of a vehicle with double-track motion with a non-linear tire mode. RSC is designed on the basis of the same method as adopted for the MPC controller to achieve a fair comparison. Then, three test cases are built in CarSim-Simulink joint platform. Specifically, the verification test is used to test the tracking accuracy of MPC and RSC controller under well road conditions. Besides, the double lane change test with low road adhesion is designed to find the maximum velocity that both controllers can carry out while guaranteeing stability. Furthermore, an extreme curve test is built where the road adhesion changes suddenly, in order to test the performance of both controllers under extreme conditions. Finally, the advantages and disadvantages of MPC and RSC under different scenarios are also discussed.
\end{abstract}

Keywords: Trajectory tracking, Automated vehicles, Model predictive control, Robust $H_{\infty}$ state feedback control

\section{Introduction}

The increasing demands on mobility, efficiency and safety have extremely promoted the development of intelligent transportation system (ITS) in recent years [1-4]. As one of the most promising technologies of automotive industry, automated vehicles (AVs) with the improved security, has great potential applications in many fields and has attracted from both industry and academic communities [5-7], many attentions. Generally, the typical architecture of an AV system mainly incorporates two parts [8, 9], namely, perception system and decision-making. Each

\footnotetext{
${ }^{*}$ Correspondence: tangx10923@cqu.eud.cn

${ }^{1}$ College of Mechanical and Vehicle Engineering, Chongqing University,

Chongqing 400000, China

Full list of author information is available at the end of the article
}

of them is, in general, divided into many subsystems. Trajectory tracking control is a fundamental issue for AVs, which is devoted to track a predefined path and velocity profiles accurately and the errors (i.e. the lateral offset, heading and velocity errors) need to converge to zero during the manipulating process [10].

In recent years, much research work on trajectory tracking have been studied [11-14] and the main challenges of achieving accurate trajectory tracking lie in the following aspects: (1) non-holonomic property and multi-constrains of AGVs; (2) trade-off between vehicle model accuracy and computation efficiency; (3) uncertainties and time-varying parameters of vehicle dynamic model; (4) external disturbances of complex driving environment. Besides, the interaction between road and tires is also an important source of coupling. According 
to the vehicle dynamics, it's known that the maximal longitudinal and lateral tire force are determined based on the friction ellipse under certain road conditions [15]. Furthermore, for most existing literatures of trajectory tracking, the bicycle vehicle model which describes longitudinal, lateral and yaw motion is usually adopted for the controller design. However, owing to the yaw motion caused by wheels steering, dynamic and kinematic couplings will put huge impact on the controllers' performance [16]. From literatures, model predictive control (MPC) and robust $H_{\infty}$ control are two effective techniques to address the problems mentioned above [17-23].

Recently, MPC has become one of the most popular optimal control methods due to its well performance in processing multi-constrained linear or non-linear system $[24,25]$, and it is easy to be used at different levels of the process control structures $[18,26]$. Owing to its advantages, MPC has been widely implemented in autonomous industry including trajectory tracking issues. The trajectory controller is designed by taking the tracking task as a multi-constrained model predictive control (MMPC) issue $[27,28]$. On the other hand, robust $H_{\infty}$ control also has certain advantages in handling the presence of model uncertainties and external disturbances, which results from variations of environment parameters as well as the vehicle states. Ref. [29] managed to devise a robust $H_{\infty}$ path following control strategy for AVs with considering signal transmission delay and data dropouts. In addition, the time-varying parameters, steering system backlashtype hysteresis, and uncertainties of nonlinear tire model were also considered in Refs. [30-32].

Though MPC and robust $H_{\infty}$ control are two effective strategies in tackling trajectory tracking issues, they show different features. Therefore, the objective of this paper is to show the difference between MPC and robust $H_{\infty}$ state feedback control (RSC) under different driving conditions. To the best of the authors' knowledge, there is no comparative study of MPC and RSC techniques for trajectory tracking. Both MPC and RSC controllers are designed based on the same longitudinal-yawlateral motions of a single-track vehicle with a linear tire model to achieve a fair comparison. The first key part of this paper is to design appropriate test cases to compare the difference between MPC and RSC controller. We re-emphasize that the main objective of this paper is a comparative study of path-following control employing different manoeuvres. Thus, the second part is to find the maximum velocity that both controllers can execute and to give corresponding reasons, which can give guidance for researchers to design controllers.

The rest of the paper are organized as follows. In Section 2 , the trajectory tracking error model and 3-DOF vehicle dynamics model are deduced. In Section 3, the controllers based on MPC and RSC are designed to track expected trajectory. Then, the performance of MPC and RSC controllers is demonstrated in Section 4. Finally, the conclusions are illustrated in Section 5.

\section{Trajectory Tracking Model}

The trajectory tracking model is shown in Figure 1 and the definition of parameters used in the design of controllers is listed in Table 1.

At any given time, it's assumed that the reference trajectory is given by a tuple $\left[x_{r}, y_{r}, \varphi_{r}, v_{r}\right]^{\mathrm{T}}$ and subscript $r$ represents reference profiles. From Figure 1, the lateral offset $e_{y}$ can be calculated by:

$$
e_{y}=\left(x-x_{r}\right) \sin \left(\varphi_{r}\right)-\left(y-y_{r}\right) \cos \left(\varphi_{r}\right) .
$$

The heading error $e_{\varphi}$ and velocity error $e_{v}$ are defined as follows

$$
\left\{\begin{array}{l}
e_{\varphi}=\varphi-\varphi_{r} \\
e_{v}=v_{x}-v_{r} .
\end{array}\right.
$$

Given the road curvature $C_{R}$, the desired yaw rate is obtained:

$$
\dot{\varphi}_{r}=v_{x} C_{R} .
$$

Besides, the derivatives of CG's position can be obtained from the kinematic relationship:

$$
\left\{\begin{array}{l}
\dot{x}=v_{x} \cos (\varphi)-v_{y} \sin (\varphi), \\
\dot{y}=v_{x} \sin (\varphi)+v_{y} \cos (\varphi) .
\end{array}\right.
$$

Thus, combined Eq. (1) with Eqs. (2)-(4), we can get

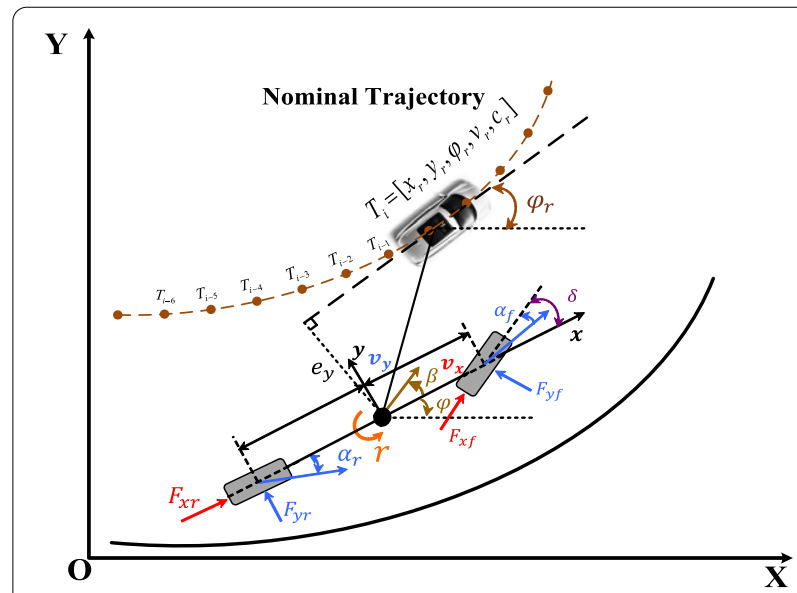

Figure 1 Schematic diagram of trajectory tracking model and vehicle dynamic model 
Table 1 Symbol and definition of the tracking system

\begin{tabular}{lll}
\hline Parameters & Unit & Description \\
\hline$x / y$ & $\mathrm{~m}$ & Coordinates of center of gravity (CG) \\
$\varphi$ & $\mathrm{rad}$ & Yaw angle of vehicle body (in inertial coordinates system, OXY) \\
$r$ & $\mathrm{rad} / \mathrm{s}$ & Yaw rate of vehicle body \\
$v_{x} / V_{y}$ & $\mathrm{~m} / \mathrm{s}$ & Longitudinal/lateral velocity (in local coordinates system, oxy) \\
$e_{y}$ & $\mathrm{~m}$ & Offset of CG from the reference point \\
$e_{\varphi}$ & $\mathrm{rad}$ & Orientation error of yaw angle with respect to the reference trajectory \\
$e_{V}$ & $\mathrm{~m} / \mathrm{s}$ & Error between the current and the desired longitudinal velocity \\
$m$ & $\mathrm{~kg}$ & Vehicle mass \\
$I_{z}$ & $\mathrm{~kg} \cdot \mathrm{m}^{2}$ & Yaw moment of inertia of the vehicle \\
$I_{f} / I_{r}$ & $\mathrm{~m}$ & Distance from CG to the front/real axle \\
$F_{x f} / F_{x r}$ & $\mathrm{~N}$ & Longitudinal tire force of the front/rear wheel \\
$F_{y f} / F_{y r}$ & $\mathrm{~N}$ & Lateral tire force of the front/rear wheel \\
$C_{f} / C_{r}$ & $\mathrm{~N} / \mathrm{rad}$ & Cornering stiffness of the front/real wheel \\
$\alpha_{f} / \alpha_{r}$ & $\mathrm{rad}$ & Slip angle of the front/ rear wheel \\
$F_{x}$ & $\mathrm{~N}$ & Generalized longitudinal force \\
$\delta_{f}$ & $\mathrm{rad}$ & Front wheel steering angle \\
$C_{R}$ & $\mathrm{~m}{ }^{-1}$ & Road curvature \\
\hline
\end{tabular}

$$
\left\{\begin{array}{l}
\dot{e}_{y}=\dot{x} \sin \left(\varphi_{r}\right)-\dot{y} \cos \left(\varphi_{r}\right)=v_{x} \sin \left(e_{\varphi}\right)+v_{y} \cos \left(e_{\varphi}\right), \\
\dot{e}_{\varphi}=r-v_{x} C_{R} \\
\dot{e}_{v}=\dot{v}_{x}-\dot{v}_{r} .
\end{array}\right.
$$

The schematic diagram of single-track vehicle model coupled 3-DOF vehicle dynamic model is also shown in Figure 1. According to Newton's laws, the vehicle dynamics in the yaw plane can be described by the following differential equations:

$$
\begin{cases}m\left(\dot{v}_{x}-r v_{y}\right) & =F_{x f}+F_{x r} \cos \delta_{f}=F_{x}, \\ m\left(\dot{v}_{y}+v_{x} r\right) & =F_{y f}+F_{y r} \\ I_{z} \dot{r} & =l_{f} F_{y f}-l_{r} F_{y r},\end{cases}
$$

where $F_{x}$ means the generalized longitudinal force of AVs including wind drag and tire rolling resistance. $F_{x f}$ and $F_{x r}$ are the longitudinal force in front wheels and rear wheels, respectively, $\delta_{f}$ is front wheel steering angle.

The front and rear lateral force $F_{y f}$ and $F_{y r}$ are the function of tire slip angles that can be calculated as follows:

$$
F_{y f}=C_{f} \alpha_{f}, F_{y r}=C_{r} \alpha_{r}
$$

where $\alpha_{f}$ and $\alpha_{r}$ denote the wheel slip angles, which can be calculated as follows:

$$
\alpha_{f}=\delta_{f}-\frac{l_{f} r+v_{y}}{v_{x}}, \alpha_{r}=\frac{l_{r} r-v_{y}}{v_{x}} .
$$

Therefore, combined Eq. (6) with Eqs. (7), (8), then

$$
\left\{\begin{array}{l}
\dot{v}_{y}=a_{11} v_{y}+a_{12} r+b_{1} \delta_{f}, \\
\dot{r}=a_{21} v_{y}+a_{22} r+b_{2} \delta_{f}, \\
\dot{e}_{y}=v_{x} \sin e_{\varphi}+v_{y} \cos e_{\varphi}, \\
\dot{e}_{\varphi}=r-C_{R}\left(e_{v}+v_{x r}\right), \\
\dot{e}_{v}=r v_{y}-\dot{v}_{x r}+\frac{F_{x}}{m}
\end{array}\right.
$$

where

$$
a_{11}=-\frac{\left(C_{f}+C_{r}\right)}{m v_{x}}, a_{12}=-v_{x}-\frac{\left(l_{f} C_{f-} l_{r} C_{r}\right)}{m v_{x}},
$$

$a_{21}=-\frac{\left(l_{f} C_{f-} l_{r} C_{r}\right)}{I_{z} v_{x}}, a_{22}=-\frac{\left(l_{f}^{2} C_{f} l_{r}^{2} C_{r}\right)}{I_{z} v_{x}}$

$b_{1}=\frac{C_{f}}{m}, b_{2}=\frac{l_{f} C_{f}}{I_{z}}$.

To facilitate the controller design, rewrite the Eq. (9) to form the following linear state-space equation, which can be represented as:

$$
\left\{\begin{array}{l}
\dot{x}(t)=A(t) x(t)+B(t) u(t)+D(t), \\
y(t)=C(t) x(t)
\end{array}\right.
$$


$A(t)=\left[\begin{array}{cccccc}a_{11} & a_{12} & 0 & & 0 & 0 \\ a_{21} & a_{22} & 0 & & 0 & 0 \\ 1 & 0 & 0 & v_{x} & 0 \\ 0 & 1 & 0 & 0 & -C_{R} \\ r & 0 & 0 & 0 & 0\end{array}\right]$,
with
$B(t)=\left[\begin{array}{cc}0 & b_{1} \\ 0 & b_{2} \\ 0 & 0 \\ 0 & 0 \\ 1 / m & 0\end{array}\right], D(t)=\left[\begin{array}{l}0 \\ 0 \\ 0 \\ -C_{R} v_{x} \\ -\dot{v}_{r}\end{array}\right], \quad C(t)=\left[\begin{array}{lllll}0 & 1 & 0 & 0 & 0 \\ 0 & 0 & 1 & 0 & 0 \\ 0 & 0 & 0 & 1 & 0 \\ 0 & 0 & 0 & 0 & 1\end{array}\right]$,

where the state variables is $x(t)=\left[v_{y}, r, e_{y}, e_{\varphi}, e_{v}\right]^{\mathrm{T}}$ and the output variables is $y(t)=\left[r, e_{y}, e_{\varphi}, e_{v}\right]^{\mathrm{T}}$. The control input is $u(t)=\left[F_{x}, \delta_{f}\right]^{\mathrm{T}}$ and $D(t)$ means disturbance.

\section{Control Law Allocation}

In the previous section, the trajectory tracking model has been established. Then, in this section, the trajectory tracking controller will be designed based on MPC and robust theory, respectively. Specifically, both controllers will be built with model described in Eq. (10) to compare the differences between them.

\subsection{Trajectory Tracking Controller Based on MPC}

\subsubsection{Formulation of Augmented System}

As analyzed above, the trajectory tracking task can be taken as a predictive control problem with multi-constraints. Note that the model described in Eq. (10) is a continuous-time system. To facilitate MPC controller design, the continuous-time system should be transformed into a discrete state-space mode with the fixed sampling period. Here, the zero-order hold $(\mathrm{ZOH})$ method is applied and the Eq. (10) can be converted as:

$$
\left\{\begin{array}{l}
x(k+1)=A_{d} x(k)+B_{d} u(k)+D(k), \\
y(k)=C_{d} x(k),
\end{array}\right.
$$

where $A_{d} \in R^{5 \times 5}, B_{d} \in R^{5 \times 2}$ and $C_{d} \in R^{4 \times 5}$ are the system coefficient matrices and $k$ means the time step.

Furthermore, to achieve a better control performance, the state vector $x(k)$ and the increment input $\Delta u(k)$ are usually coupled in an augmented vector, which can be represented as $\tilde{x}(k)=[x(k), \Delta u(k)]^{\mathrm{T}}$. And the control input can be calculated by

$$
u(k)=u(k-1)+\Delta u(k) .
$$

Thus, the system Eq. (11) can be rewritten as the following equations:

$$
\left\{\begin{array}{l}
\tilde{x}(k+1)=\tilde{A}(k) \tilde{x}(k)+\tilde{B}(k) \Delta u(k)+\tilde{D}(k), \\
\tilde{y}(k)=\tilde{C}(k) \tilde{x}(k),
\end{array}\right.
$$

where $\quad \tilde{A}(k)=\left[\begin{array}{cc}A_{d} & B_{d} \\ 0_{2 \times 5} & I_{2}\end{array}\right], \quad \tilde{B}(k)=\left[\begin{array}{c}B_{d} \\ I_{2}\end{array}\right] \quad$ and $\tilde{C}(k)=\left[\begin{array}{ll}C_{d} & 0_{4 \times 2}\end{array}\right]$.

The sequence of future incremental inputs and disturbances at time step $k$ are denoted as $\Delta U(k)$ and $\bar{D}(k)$, respectively.

$$
\left\{\begin{aligned}
\Delta U(k) & =\left[\Delta u(k), \Delta u(k+1), \cdots, \Delta u\left(k+N_{c}-1\right)\right]^{\mathrm{T}}, \\
\bar{D}(k) & =\left[\tilde{D}(k), \tilde{D}(k+1), \cdots, \tilde{D}\left(k+N_{p}-1\right)\right]^{\mathrm{T}},
\end{aligned}\right.
$$

where $N_{p}$ is the prediction horizon and $N_{c}$ means the control horizon.

Therefore, the predicted output can be calculated by the following formulations:

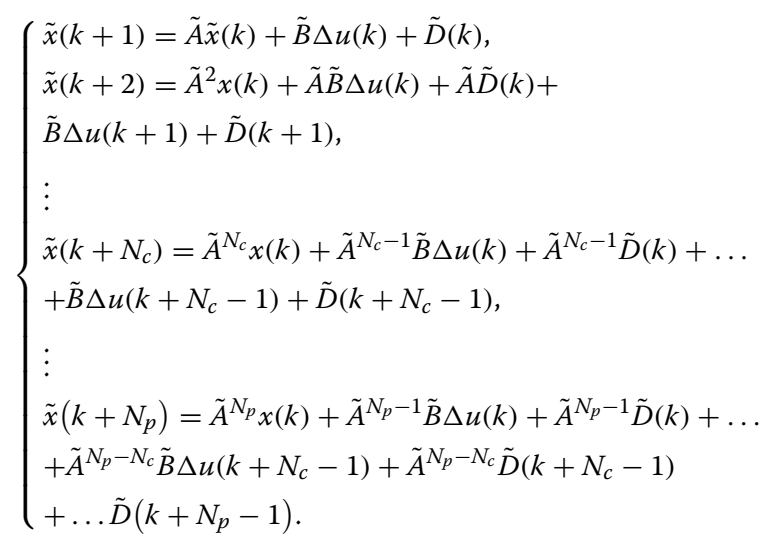

Here, define the predicted outputs of the predictive state-space model at time step $k$ as:

$$
Y(k)=\left[\tilde{y}(k+1), \tilde{y}(k+2), \cdots, \tilde{y}\left(k+N_{p}\right)\right]^{\mathrm{T}} .
$$

Denote the performance outputs over the prediction horizon $N_{p}$ as a compact matrix form:

$$
Y(k)=\Gamma \tilde{x}(k)+\Theta \Delta U(k)+\xi \bar{D}(k),
$$

with $\Gamma=\left[\tilde{C} \tilde{A}, \tilde{C} \tilde{A}^{2}, \cdots \tilde{C} \tilde{A}^{N_{c}}, \cdots, \tilde{C} \tilde{A}^{N_{p}}\right]^{\mathrm{T}}$, 


$$
\begin{aligned}
& \Theta=\left[\begin{array}{cccc}
\tilde{C} \tilde{B} & 0 & \cdots & 0 \\
\tilde{C} \tilde{A} \tilde{B} & \tilde{C} \tilde{B} & \cdots & \vdots \\
\vdots & \vdots & \cdots & 0 \\
\tilde{C} \tilde{A}^{N_{c}-1} \tilde{B} & \tilde{C} \tilde{A}^{N_{c}-2} \tilde{B} & \cdots & \tilde{C} \tilde{B} \\
\vdots & \vdots & \ddots & \vdots \\
\tilde{C} \tilde{A}^{N_{c}-1} \tilde{B} & \tilde{C} \tilde{A}^{N_{c}-2} \tilde{B} & \cdots & \tilde{C} \tilde{A}^{N_{p}-N_{c} \tilde{B}}
\end{array}\right], \\
& \xi=\left[\begin{array}{cccc}
\tilde{C} & 0 & \cdots & 0 \\
\tilde{C} \tilde{A} & \tilde{C} & \cdots & 0 \\
\tilde{C} \tilde{A}^{2} & \tilde{C} \tilde{A} & \cdots & 0 \\
\vdots & \vdots & \ddots & \vdots \\
\tilde{C} \tilde{A}^{N_{p}-1} & \tilde{C} \tilde{A}^{N_{p}-2} & \cdots & \tilde{C}
\end{array}\right]
\end{aligned}
$$

\subsubsection{Formulation of Trajectory Tracking Problem Using MPC}

The aim of MPC-based trajectory tracking controller is to make the predicted outputs as close as possible to the reference trajectory within the predictive horizon, and the reference trajectory $Y_{r}(k)$ is assumed to remain unchanged during current optimization window. The reference signals are described as:

$$
Y_{r}(k)=\left[\tilde{y}_{r}(k+1), \tilde{y}_{r}(k+2), \cdots, \tilde{y}_{r}\left(k+N_{p}\right)\right]^{\mathrm{T}} .
$$

Thus, a typical tracking accuracy and control smoothness-oriented cost function over the predictive horizons is defined:

$$
\begin{aligned}
& \mathcal{L}(x, u(k-1), U(k)) \\
& \quad=\min _{\Delta U_{k}}\left\{\left[Y_{r}(k)-Y(k)\right]^{\mathrm{T}} \overline{\mathrm{Q}}\left[Y_{r}(k)-Y(k)\right]+\Delta U^{\mathrm{T}}(k) \bar{R} \Delta U(k)\right\},
\end{aligned}
$$

where diagonal matrices $\bar{Q}$ and $\bar{R}$ are the positive definite weight matrices (i.e., $\bar{Q}>0$ and $\bar{R}>0$ ) which can be regulated to achieve desired closed-loop performance. The first item in cost function reflects the tracking error between the predictive outputs and the reference trajectory. The second one refers to the penalty on the control inputs that is to make the control process smoother.

On the other hand, vehicles have many inherent physical limitations. These limitations on the capacity of control actuators result in the hard constrains of the tracking system. Besides, there are also some restrictions imposed on output variables due to environment conditions (i.e., road boundary, speed limit, etc.). Here, the constrains for this trajectory tracking problem are specified as:

$$
\left\{\begin{array}{l}
\Delta U_{\min }(k) \leq \Delta U(k) \leq \Delta U_{\max }(k) \\
U_{\min }(k) \leq G \Delta U+F u(k-1) \leq U_{\max }(k) \\
Y_{\min } \leq Y(k) \leq Y_{\max }
\end{array}\right.
$$

with

$$
G=\left[\begin{array}{ccccc}
1 & 0 & \cdots & 0 & 0 \\
1 & 1 & \cdots & 0 & 0 \\
1 & 1 & \ddots & \vdots & \vdots \\
1 & 1 & \cdots & 1 & 0 \\
1 & 1 & \cdots & 1 & 1
\end{array}\right]_{N_{c} \times N_{c}} F=\left[\begin{array}{l}
1 \\
1 \\
\vdots \\
1 \\
1
\end{array}\right]_{N_{c} \times 1}
$$

where $\quad \Delta U_{\min }, \Delta U_{\max }, U_{\min }, U_{\max } \in R^{2 N_{c} \times 1}, \quad Y_{\min }$, $Y_{\max } \in R^{4 \times 1}$ and $u(k-1)$ is the control inputs at time $k-1$.

Then, combined Eq. (19) with Eq. (20), the trajectory tracking problem is converted to solve the following optimal problem with multi-constrains:

$$
\begin{aligned}
& \mathcal{L}(x, u(k-1), U(k))= \\
& \min _{\Delta U_{k}}\left\{\left[Y_{r}(k)-Y(k)\right]^{\mathrm{T}} \bar{Q}\left[Y_{r}(k)-Y(k)\right]+\Delta U^{\mathrm{T}}(k) \bar{R} \Delta U(k)\right\}, \\
& \text { s.t., }\left\{\begin{array}{l}
\Delta U_{\min } \leq \Delta U \leq \Delta U_{\max }, \\
U_{\min } \leq G \Delta U+F u(k-1) \leq U_{\max }, \\
Y_{\min } \leq Y(k) \leq Y_{\max } .
\end{array}\right.
\end{aligned}
$$

It's found that the optimization problem Eq. (21) is based on linear system and the cost function is convex quadratic, which means it's can be remade as quadratic program $(\mathrm{QP})$ issues if the constrains are convex too. The problem described in Eq. (21) needs to be translated into standard form of quadratic problems:

$$
\begin{array}{r}
\mathcal{L}^{\prime}(x, u(k-1), U(k))=\min _{\Delta U_{k}}\left\{\frac{1}{2} \Delta U^{\mathrm{T}}(k) G_{k} \Delta U(k)+H^{\mathrm{T}} \Delta U(k)\right\} \\
\text { s.t., }\left\{\begin{array}{l}
\Delta U_{\min } \leq \Delta U \leq \Delta U_{\max }, \\
U_{\min } \leq G \Delta U+F u(k-1) \leq U_{\max }, \\
Y_{\min } \leq Y(k) \leq Y_{\max },
\end{array}\right.
\end{array}
$$

where $G_{k}=2\left(\Theta^{\mathrm{T}} \overline{\mathrm{Q}} \Theta+\overline{\mathrm{R}}\right), H=\Theta^{\mathrm{T}} \overline{\mathrm{Q}}\left(\Gamma \tilde{x}-Y_{r}+\xi \bar{D}\right)$.

Then, at time step $k$, the above quadratic problem can be solved (i.e., quadprog function in Matlab) and will get a solution $\Delta U_{k}^{*}$. Once the solution is obtained, the first element of solution vector in vector, namely, $\Delta u_{k}^{*}$ will be used and the optimal control inputs at this time step is computed as:

$$
u(k)=u(k-1)+\Delta u_{k}^{*}
$$

Similarly, at next time step $k+1$, new input measurements and updated system states produce new quadratic problem that needs to solve. In conclusion, by solving a quadratic problem at each time step, the MPC controller will get an optimal input for next time step. 


\subsection{Trajectory Tracking Controller Based on RSC 3.2.1 Preliminary Knowledge of RSC}

We re-emphasize that the main objective of this paper is a comparative study of different control strategies. Both MPC-based and RSC-based tracking controllers use standard design methods, and specifically, the RSC-based tracking controller design mainly adopts the theory applied in Refs. [10, 21]. The design of RSC is also based on the linearized vehicle model Eq. (10) and the objective function is defined as follows:

$$
\int_{0}^{t} y^{\mathrm{T}}(t) y(t) \mathrm{d} t \leq \gamma^{2} \int_{0}^{t} D^{\mathrm{T}}(t) D(t) \mathrm{d} t
$$

where the main task is to obtain a control law that can make the closed-loop system satisfy the asymptotically stability and fulfill the $H_{\infty}$ performance index $\gamma$ that attenuate the effect of the external disturbance $D(t)$.

Before the controller design, some essential lemmas and theorems also need to be introduced. Consider a polytopic linear parameter varying (LPV) system, which is described as follows:

$$
\left\{\begin{array}{l}
\dot{x}(t)=A_{0}(q) x(t)+B_{0}(q) u(t), \\
y(t)=C_{0}(q) x(t) .
\end{array}\right.
$$

It's equal to the following equations:

$$
\left\{\begin{aligned}
\dot{x}(t) & =\sum_{i=1}^{n} q_{i}\left[A_{0 i} x(t)+B_{0 i} u(t)\right], \\
y(t) & =\sum_{i=1}^{n} q_{i} C_{0 i} x(t), \\
\sum_{i=1}^{n} q_{i} & =1, q_{i} \geq 0,
\end{aligned}\right.
$$

where the $A_{0 i}, B_{0 i}$ and $C_{0 i}$ are the values of $A_{0}(q), B_{0}(q)$ and $C_{0}(q)$ at the vertex $q_{i}$ of the parameters polytope.

Theorem 1 Given a positive scalar $\gamma_{0}$, the system described in Eq. (26) is asymptotically stable and meets the $H_{\infty}$ performance index in Eq. (24), if and only if there exists $n$ symmetric definite matrices $P_{i}$ and a matrix $S_{0}$ satisfying the following conditions

$$
\left[\begin{array}{cccc}
A_{0 i} S_{0}+S_{0}^{\mathrm{T}} A_{0 i}^{\mathrm{T}} & S_{0}^{\mathrm{T}} B_{0 i}^{\mathrm{T}} & C_{0 i} & P_{i}-S_{0}^{\mathrm{T}} \\
* & -\gamma_{0} I & 0 & 0 \\
* & * & -\gamma_{0} I & 0 \\
* & * & * & 0
\end{array}\right]<0, i=1,2, \cdots, n .
$$

The proof of this theorem can be found in Ref. [21]. The RSC-based controller design is on account of linear matrix inequality (LMI) method. So far, the preliminary knowledge has been prepared sufficiently for the RSCbased controller design.

\subsubsection{Formulation of Trajectory Tracking Problem}

Similar to MPC-based controller, the constrains imposed on inputs should be taken into consideration [33]. Define Lyapunov equation as follows:

$$
V(t)=x(t)^{\mathrm{T}} P x(t) .
$$

And it's assumed that the following conditions are satisfied

$$
\begin{aligned}
& x(t)^{\mathrm{T}} P x(t)<\eta, \\
& u(t)=K x(t),
\end{aligned}
$$

where $K$ is the gain of the controller and $\eta$ is a given constant. The maximum inputs are denoted as $u_{\max }$, then,

$$
\begin{aligned}
& \max \|u(t)\|^{2}=\max \left\|x^{\mathrm{T}}(t) K^{\mathrm{T}} K x(t)\right\| \\
&=\max \left\|x^{\mathrm{T}}(t) P^{\frac{1}{2}} P^{-\frac{1}{2}} K^{\mathrm{T}} K P^{-\frac{1}{2}} P^{\frac{1}{2}} x(t)\right\| \\
&<\eta \theta_{\max }\left(P^{-\frac{1}{2}} K^{\mathrm{T}} K P^{-\frac{1}{2}}\right) \leq u_{\max } I,
\end{aligned}
$$

where $\theta_{\max }$ is the largest eigenvalue of the matrix $\left(P^{-\frac{1}{2}} K^{\mathrm{T}} K P^{-\frac{1}{2}}\right)$.

Based on Schur complement lemma [34] and Eq. (31), the following conditions can be obtained,

$$
\left[\begin{array}{cc}
-I & \sqrt{\eta} K \\
\sqrt{\eta} K & -u_{\max }^{2} P
\end{array}\right] \leq 0 .
$$

For the system depicted by Eq. (10), it can be found that time-varying parameters $v_{x}, \frac{1}{v_{x}}, r$ and $C_{R}$ are coupled in the system matrices. These parameters need to be processed before carrying out the design of RSC control law. Here, the polytope model method in Ref. [10] is applied to cover the rang of time-varying parameters. Specifically, in this system, the longitudinal velocity is usually bounded. It can be assumed that $v_{x}$ varies in the range of $\left[v_{x \min }, v_{x \max }\right], \frac{1}{v_{x}}$ is bound in the range of $\left[\frac{1}{v_{x \max }}, \frac{1}{v_{x \min }}\right]$ and $v_{x \min }, v_{x \max }$ denote the minimum and maximum values of longitudinal velocity. Similarly, the same assumptions are imposed on $r$ and $C_{R}$, namely, $r$ and $C_{R}$ vary in the range of $\left[r_{\max }, r_{\min }\right]$ and $\left[C_{R \min }, C_{R \max }\right]$, respectively. Therefore, the time-varying parameters $v_{x}, \frac{1}{v_{x}}, r$ and $C_{R}$ can be expressed by a linear combination of the vertices as follows: 


$$
\left\{\begin{array}{l}
v_{x}=\sum_{i=1}^{2} \rho_{1 i} \sigma_{1 i}, \quad \frac{1}{v_{x}}=\sum_{i=1}^{2} \rho_{2 i} \sigma_{2 i}, \\
r=\sum_{i=1}^{2} \rho_{3 i} \sigma_{3 i}, \quad C_{R}=\sum_{i=1}^{2} \rho_{4 i} \sigma_{4 i},
\end{array}\right.
$$

where $\sigma$ represents the vertices coordinates of the polytope and $\rho$ means the weighting factors, which can be denoted as:

$$
\begin{aligned}
& \left\{\begin{array}{l}
\rho_{11}=\frac{v_{x \max }-v_{x}}{v_{x \max }-v_{x \min }}, \quad \rho_{12}=\frac{v_{x}-v_{x \min }}{v_{x \max }-v_{x \min }}, \\
\rho_{21}=\frac{\frac{1}{v_{x \max }}-\frac{1}{v_{x}}}{\frac{1}{v_{x \max }}-\frac{1}{v_{x \min }}}, \rho_{22}=\frac{\frac{1}{v_{x}}-\frac{1}{v_{x \min }}}{\frac{1}{v_{x \max }}-\frac{1}{v_{x \min }}}, \\
\rho_{31}=\frac{r_{\max }-r}{r_{x \max }-r_{x \min }}, \quad \rho_{32}=\frac{r-r_{\min }}{r_{x \max }-r_{x \min }}, \\
\rho_{41}=\frac{C_{R \max }-C_{R}}{C_{R \max }-C_{R \min }}, \rho_{42}=\frac{C_{R}-C_{R \min }}{C_{R \max }-C_{R \min }},
\end{array}\right. \\
& \left\{\begin{array}{c}
\sigma_{11}=v_{x \min }, \quad \sigma_{12}=v_{x \max }, \\
\sigma_{21}=\frac{1}{v_{x \min }}, \quad \sigma_{22}=\frac{1}{v_{x \max }}, \\
\sigma_{31}=r_{\min }, \quad \sigma_{32}=r_{\max }, \\
\sigma_{41}=C_{R \min }, \quad \sigma_{42}=C_{R \max } .
\end{array}\right.
\end{aligned}
$$

Combined Eq. (10) and Eq. (33), the system plant of trajectory tracking system can be rewritten in the polytypic form as:

$$
\dot{x}(t)=\sum_{i=1}^{16} \beta_{i}\left[A_{i} x(t)+B_{i} u(t)+D_{i}(t)\right],
$$

where $\quad \beta_{1}=\bar{\beta}_{1111}, \beta_{2}=\bar{\beta}_{1112}, \cdots, \beta_{16}=\bar{\beta}_{2222}, \sum_{i=1}^{16} \beta_{i}=1 \quad$ and $\bar{\beta}_{i j m n}=\sum_{i=1}^{2} \sum_{j=1}^{2} \sum_{m=1}^{2} \sum_{n=1}^{2} \rho_{1 i} \rho_{2 j} \rho_{3 m} \rho_{4 n}$.

Define $\beta=\left[\beta_{1}, \beta_{2}^{m=1}, \cdots, \beta_{16}\right]^{\mathrm{T}}, y=\left[r, e_{y}, e_{\varphi}, e_{v}\right]^{T}$ and the LPV model of trajectory tracking system can be expressed as:

$$
\left\{\begin{array}{l}
\dot{x}(t)=A(\beta) x(t)+B(\beta) u(t)+D(t), \\
y(t)=C x(t)
\end{array}\right.
$$

with

$$
C=\left[\begin{array}{lllll}
0 & 1 & 0 & 0 & 0 \\
0 & 0 & 1 & 0 & 0 \\
0 & 0 & 0 & 1 & 0 \\
0 & 0 & 0 & 0 & 1
\end{array}\right]
$$

Then, the state-feedback controller is proposed as:

$$
u(t)=K(\beta) x(t)=\sum_{i=1}^{16} \beta_{i} K_{i} x(t),
$$

where $K_{i}$ is the control gain.
Combined the Eq. (36) with Eq. (37), the closed-loop system can be obtained:

$$
\left\{\begin{array}{l}
\dot{x}(t)=\sum_{i=1}^{16} \beta_{i}\left[\left(A_{i}+B_{i} K_{i}\right) x(t)+D_{i}(t)\right] \\
y=C x(t)
\end{array}\right.
$$

According to theorem 1 and Eq. (38), if the following condition is fulfilled, the controller will achieve the aim of ensuring system stability and $H_{\infty}$ performance index of the closed-loop system.

$$
\begin{aligned}
& {\left[\begin{array}{cccc}
\left(A_{i}+B_{i} K_{i}\right) S+S^{\mathrm{T}}\left(A_{i}+B_{i} K_{i}\right)^{\mathrm{T}} & S^{\mathrm{T}} C^{\mathrm{T}} & I & P_{i}-S^{\mathrm{T}} \\
* & -\gamma I & 0 & 0 \\
* & * & -\gamma I & 0 \\
* & * & * & 0
\end{array}\right]<0, i=1,2, \cdots, n,} \\
& {\left[\begin{array}{cc}
-I & \sqrt{\eta} K_{i} \\
\sqrt{\eta} K & -u_{\text {max }}^{2} P_{i}
\end{array}\right] \leq 0, i=1,2, \cdots, n,}
\end{aligned}
$$

where the $P_{i}$ is a symmetric positive definite matrix and $S$ is a matrix with proper dimensions. $\gamma$ represents the performance index of system in attenuating disturbance.

So far, the key to solve the trajectory tracking issue has converted to calculate the controller gain $K(\beta)$ by solving the inequality problems above. In this paper, we use the yalmip toolbox to solve this issue because it has simple syntax and is easy to use.

\section{Comparative Study of MPC-Based and RSC-Based Controllers}

In this section, three driving scenarios will be designed to compare the difference between MPC-based and RSC-based controllers. To begin with, the simulation description and section are detailed. Then, the function verification test is displayed to show that the parameters of both controllers had been tuned as optimal as possible. Finally, double lane change and extreme curve test are illustrated to compare the performance of both controllers in the harsh driving environment.

\subsection{Simulation Description and Setup}

Here, three simulation tests were designed to compare the different performance of MPC and RSC control schemes, which are carried out on the CarSim-Simulink platform. Specifically, these tests are (1) function verification, (2) double lane change manoeuvre with low adhesion, (3) curve test (curvature: 0.02) with adhesion changing abruptly. Besides, to quantify the tracking accuracy, the root mean square error (RMSE) was used, which is expressed as 
Table 2 Symbol and definition of parameters

\begin{tabular}{lll}
\hline Definition & Symbol & Value \\
\hline Vehicle mass & $m(\mathrm{~kg})$ & 1750 \\
Inertia moment of the vehicle about yaw axis & $I_{z}\left(\mathrm{~kg} \cdot \mathrm{m}^{2}\right)$ & 2500 \\
Distance of the front/rear axle from CG & $I_{f} / I_{r}(\mathrm{~m})$ & $1.24 / 1.46$ \\
Nominal cornering stiffness of front/rear wheels & $C_{f} / C_{r}(\mathrm{kN} / \mathrm{rad})$ & $60 / 60$ \\
Minimal/maximal longitudinal velocity & $V_{\min } / r_{\max }(\mathrm{m} / \mathrm{s})$ & $5 / 30$ \\
Minimal/maximal yaw rate & $r_{\min } / r_{\max }(\mathrm{rad} / \mathrm{s})$ & $-0.5 / 0.5$ \\
Minimal/maximal curvature of the path & $C_{R \min } / C_{R \max }\left(\mathrm{m}^{-1}\right)$ & $-0.02 / 0.02$ \\
& &
\end{tabular}

$$
R M S E=\sqrt{\frac{1}{M} \sum_{i=1}^{m}\left(y_{i}-y_{r}\right)^{2}},
$$

where $M$ is the number of time periods, $y_{i}$ is the measured outputs and $y_{r}$ donates reference values. The parameters of AVs and controllers used in simulation are listed in Table 2.

\subsection{Case 1: Function Verification}

In this section, the presented controllers were verified to demonstrate that the parameters of both controllers had been tuned as optimal as possible. Here, a straight line with expected velocity $25 \mathrm{~m} / \mathrm{s}$ was applied as the reference trajectory. The initial lateral, angular and velocity errors of AVs were set as $-1 \mathrm{~m}, 0,-5 \mathrm{~m} / \mathrm{s}$, respectively. Besides, the road adhesion is set as 0.85 .

As shown in Figures 2 and 3, it can be seen that MPCbased controller showed a faster response than RSCbased one and both controllers tracked the expected trajectory exactly. To be specific, the path tracking error was MPC-based controller is $0.2372 \mathrm{~m}$, which was smaller than that of RSC-based one $0.2582 \mathrm{~m}$. Besides, the velocity errors of MPC and RSC controller were $1.4520 \mathrm{~m} / \mathrm{s}$ and $1.4470 \mathrm{~m} / \mathrm{s}$, respectively. It can be concluded that in this test, MPC showed better performance that RSC in terms of tracking accuracy. The reason why it occurred was that in well driving conditions, MPC was able to predict system's future states precisely based on current states, and it takes the tracking issue as an optimal issue in current time. Owing to that the optimal issue is convex as shown in Eq. (21), of course, it would be better. On the other hand, constraints of actuators should also be considered. Here, from Figures 4 and 5, it is shown that the steer angle and longitudinal acceleration were limited in an expected range.

Overall, in this section, both controllers were verified and the parameters of them had been tuned through trial and error method, trying to ensure that both controllers can achieve well performance. In next sections,
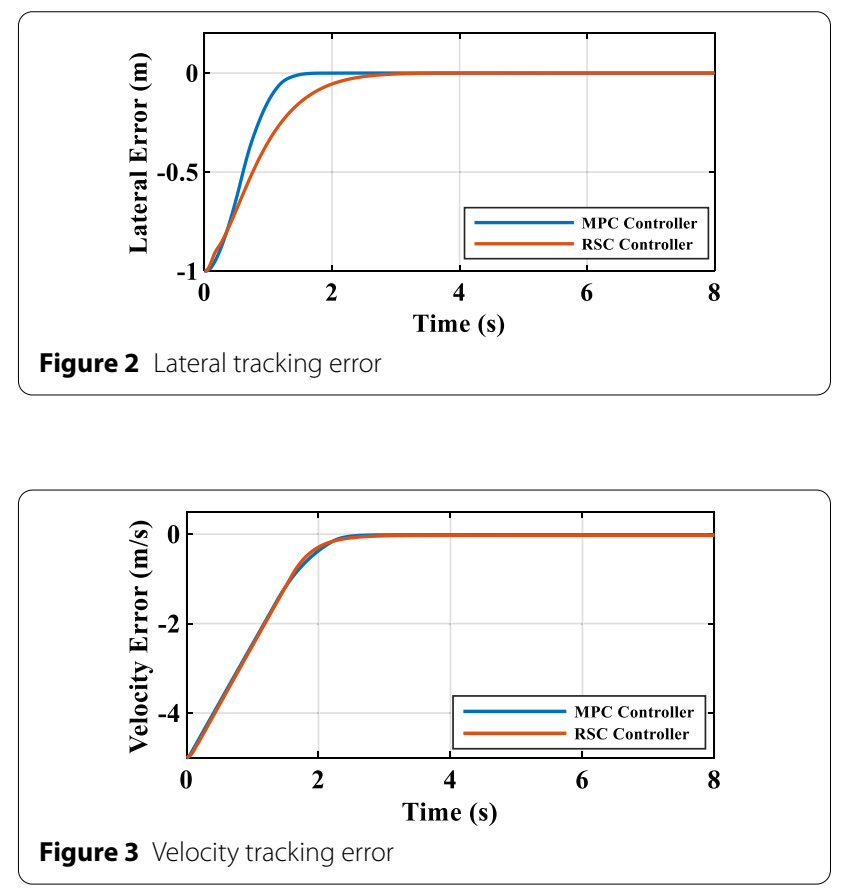

some extreme conditions were carried out based on these parameters.

\subsection{Case 2: Double Lane Change Test}

In last section, both MPC-based and RSC-based controllers were tested in well driving conditions. The results show that both controllers tracked the expected trajectory exactly.

Obviously, it's better to choose MPC controller for its accuracy. However, in this section, we were concerned with the performance of both controllers in the harsh driving environment, because some unexpected cases may occur that will put huge effects on vehicle's safety and stability.

Therefore, in this section, MPC and RSC controllers were tested in the road with low adhesion. Specifically, both controllers carried out the double lane change 


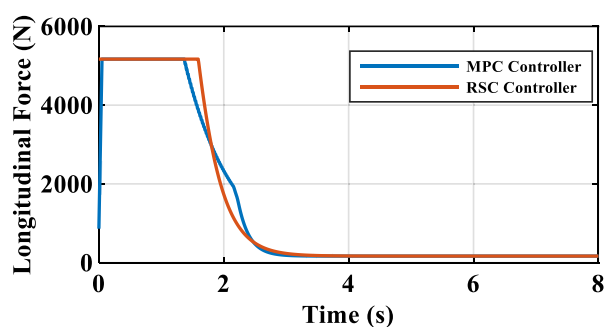

Figure 4 Command of longitudinal force

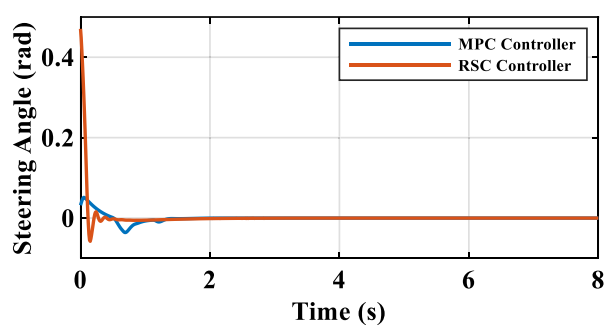

Figure 5 Command of steering angle
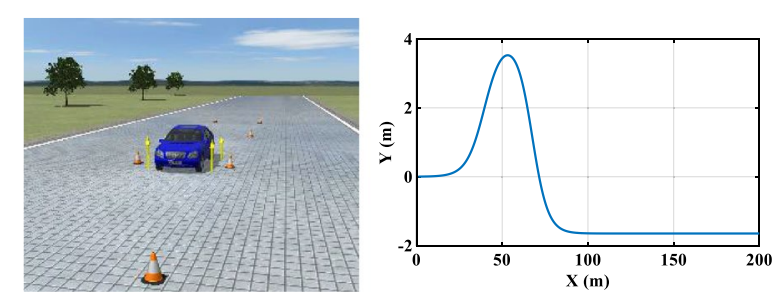

Figure 6 CarSim setup and double lane change manoeuvre

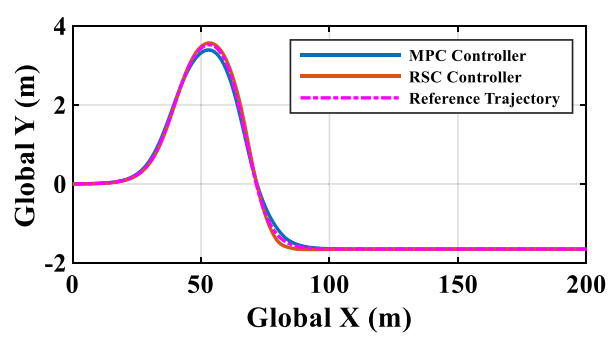

Figure $\mathbf{7}$ Comparison of trajectory when velocity was $9 \mathrm{~m} / \mathrm{s}$

manoeuvre as shown in Figure 6. Here, the road adhesion was set as 0.2 , corresponding to snow and ice road. It's known that vehicles are prone to lose lateral stability on low adhesion roads, especially when the velocity is high. Thus, the goal of this section is to find the maximum

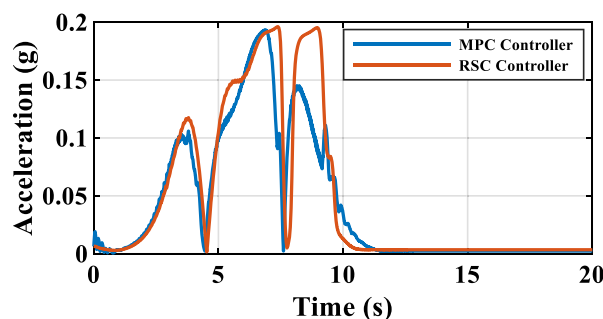

Figure 8 Acceleration information when velocity was $9 \mathrm{~m} / \mathrm{s}$

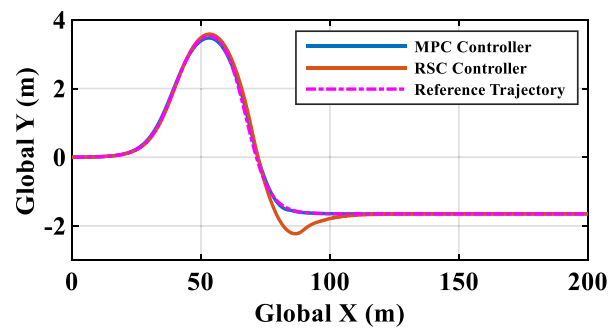

Figure $\mathbf{9}$ Comparison of trajectory when velocity was $10 \mathrm{~m} / \mathrm{s}$

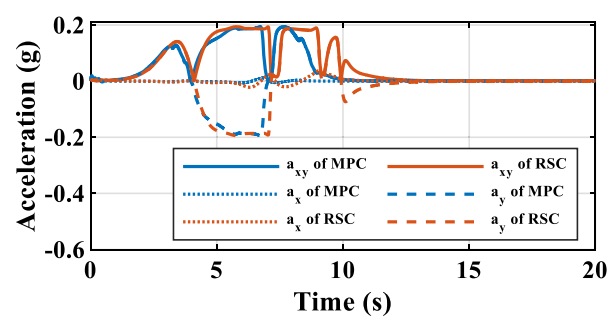

Figure 10 Acceleration information when velocity was $10 \mathrm{~m} / \mathrm{s}$

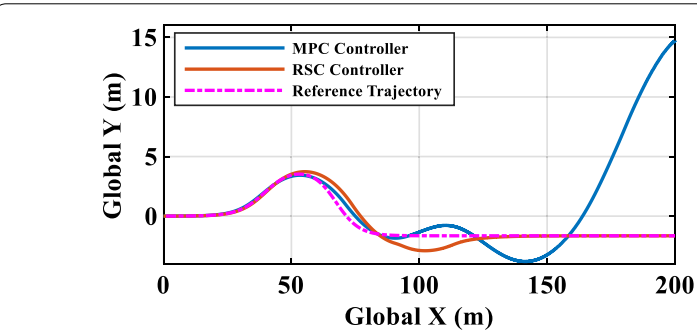

Figure 11 Comparison of trajectory when velocity was $11 \mathrm{~m} / \mathrm{s}$

velocity that both controllers can carry out with guaranteeing that the vehicle is under control. The initial lateral, angular and velocity errors of AVs were all set as 0 .

Figures 7 and 8 demonstrates the simulation results when the expected velocity was set as $9 \mathrm{~m} / \mathrm{s}$. From Figure 7, it can be found that both MPC and RSC tracked the expected trajectory well on the road with adhesion 


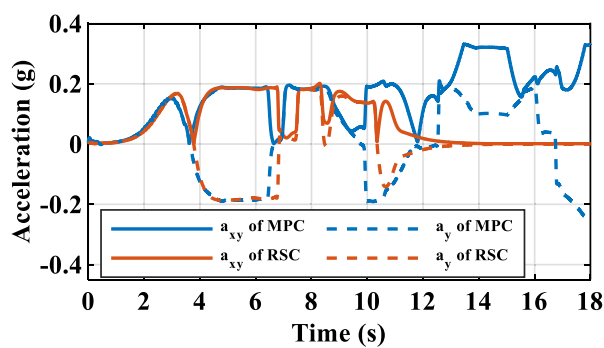

Figure 12 Acceleration information when velocity was $11 \mathrm{~m} / \mathrm{s}$

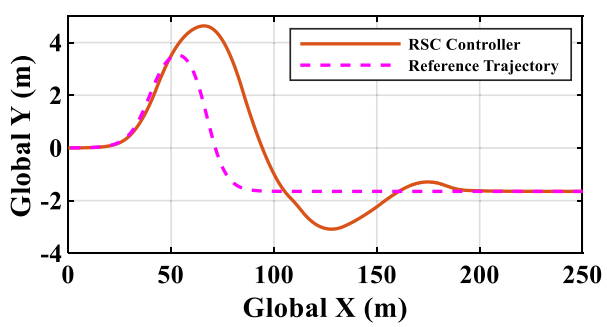

Figure 13 Trajectory of RSC when velocity was $14 \mathrm{~m} / \mathrm{s}$

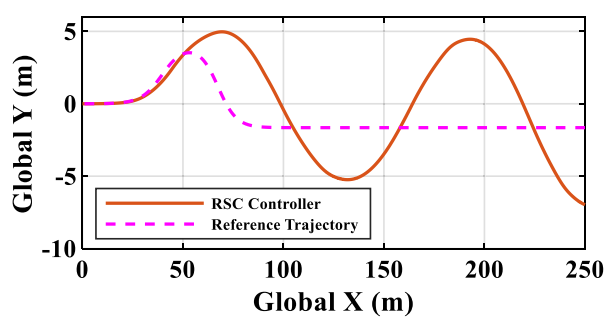

Figure $\mathbf{1 4}$ Trajectory when velocity was $15 \mathrm{~m} / \mathrm{s}$

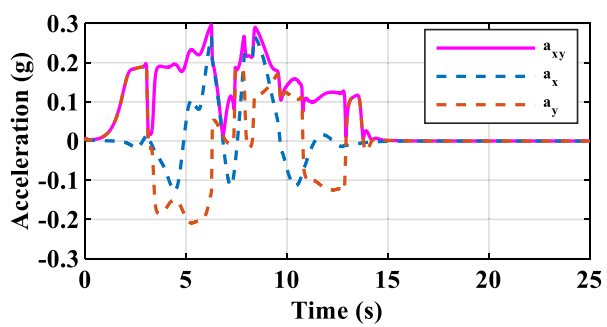

Figure 16 Acceleration information when velocity was $15 \mathrm{~m} / \mathrm{s}$

0.2. Specifically, the RMES of path tracking error for MPC and RSC were $0.0597 \mathrm{~m}$ and $0.0455 \mathrm{~m}$, respectively. Figure 8 demonstrates the variation of acceleration where the maximum value did not exceed $0.2 g$, in other words, in this case, the vehicle was under control without slipping for both controllers.

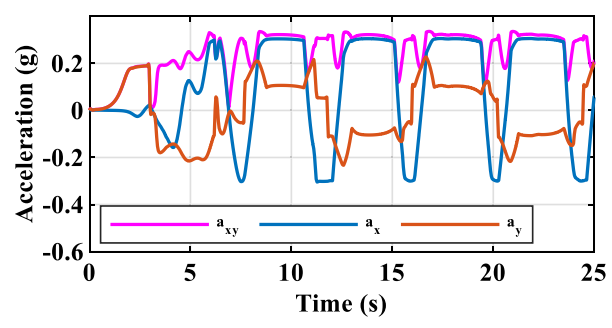

Figure 15 Acceleration information when velocity was $14 \mathrm{~m} / \mathrm{s}$
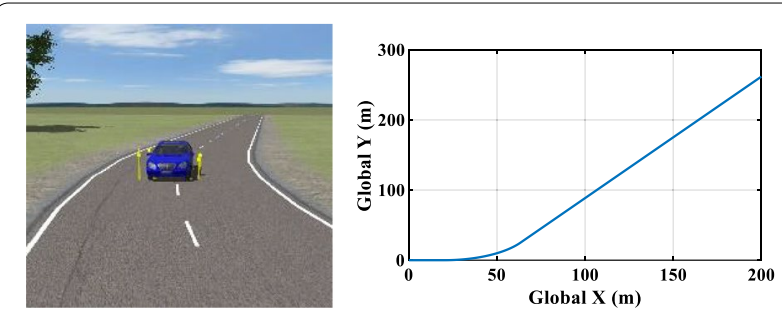

Figure 17 CarSim setup and curve manoeuvre

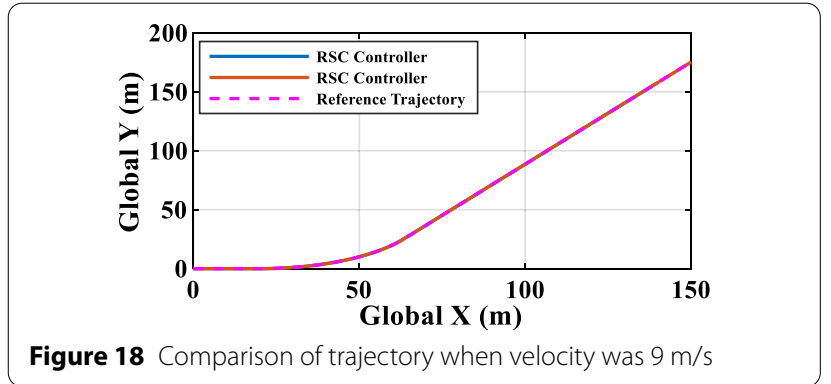

Figure 18 Comparison of trajectory when velocity was $9 \mathrm{~m} / \mathrm{s}$

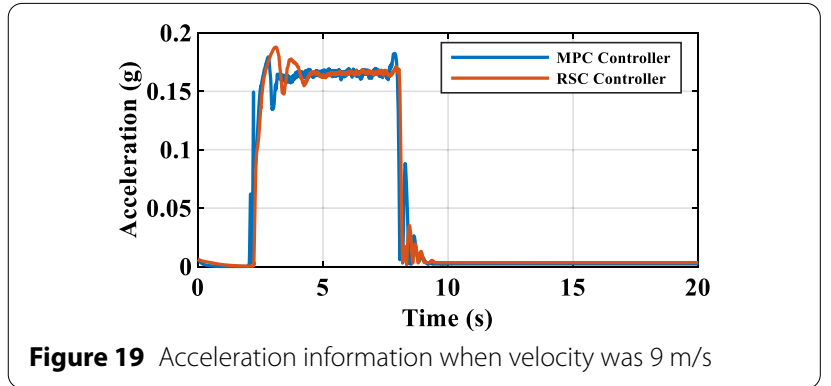

Figure 9 displays the results of MPC and RSC when the expected velocity was set as $10 \mathrm{~m} / \mathrm{s}$. In this situation, MPC showed well performance in tracking expected trajectory, where the REMS of path tracking error is $0.0342 \mathrm{~m}$.

Compared with MPC, the vehicle controlled by RSC slipped at position where $\mathrm{X}$ approximately equals to 


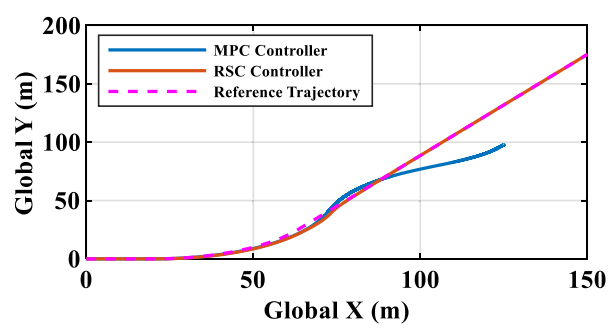

Figure $\mathbf{2 0}$ Comparison of trajectory when velocity was $10 \mathrm{~m} / \mathrm{s}$

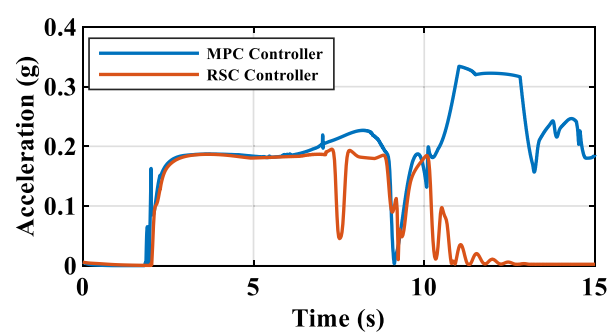

Figure 21 Acceleration information when velocity was $10 \mathrm{~m} / \mathrm{s}$

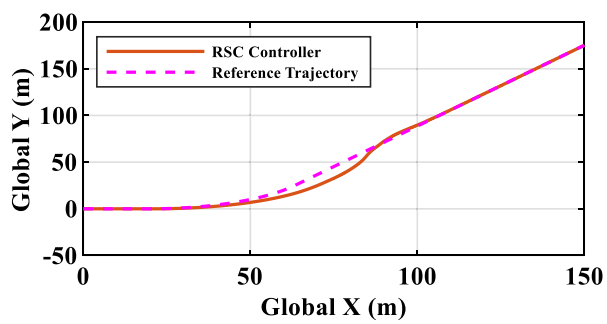

Figure 22 Trajectory of RSC when velocity was $11 \mathrm{~m} / \mathrm{s}$

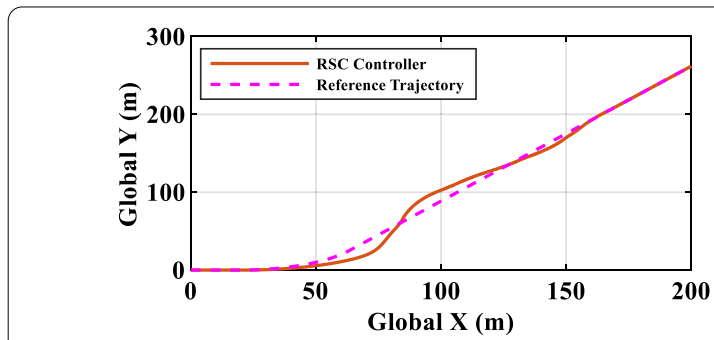

Figure 23 Trajectory of RSC when velocity was $12 \mathrm{~m} / \mathrm{s}$

$78 \mathrm{~m}$. What's the reason? From Figure 10, in this case, it can be known that the maximum acceleration equaled to $0.2 g$ approximately, which means the vehicle was under the critical states. Compared to RSC controller, the phases of MPC that acceleration approached to $0.2 \mathrm{~g}$ was short. Thus, it can be seen in Figure 9 that the vehicle controlled by RSC obviously slipped, while the vehicle

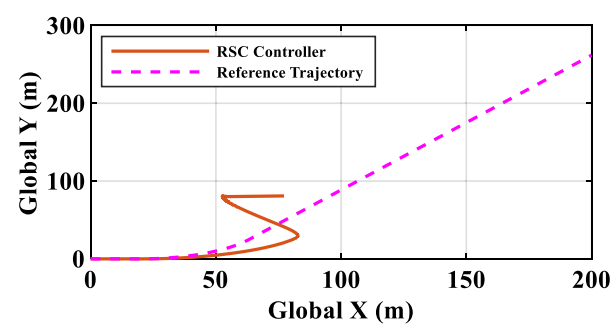

Figure 24 Trajectory of RSC when velocity was $13 \mathrm{~m} / \mathrm{s}$

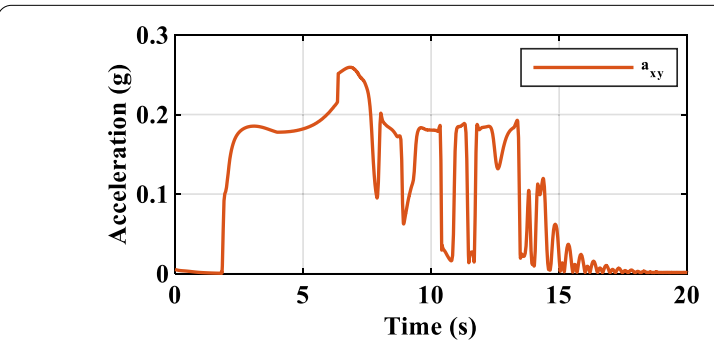

Figure 25 Acceleration information when velocity was $11 \mathrm{~m} / \mathrm{s}$

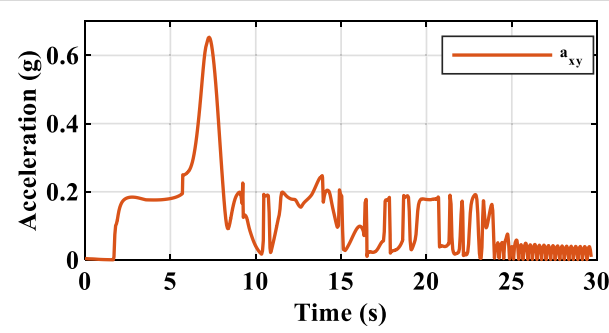

Figure 26 Acceleration information when velocity was 12 m/s

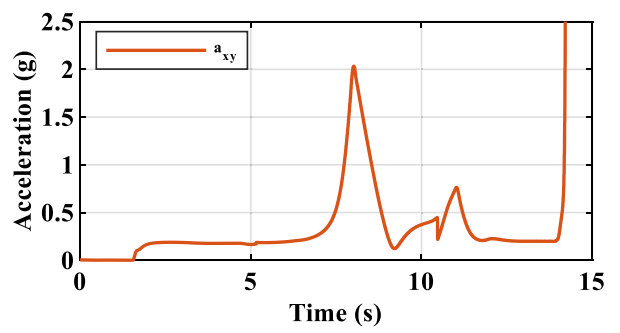

Figure $\mathbf{2 7}$ Acceleration information when velocity was 13 m/s

controlled by MPC didn't experience that. The reason why MPC shows well performance is that it can predict the future states based on current states. Note that is this case, the states MPC utilized in its prediction model was correct, in other words, the current states were still in the accepted range of the model. For RSC, due to that the gain of controller was calculated offline, so the tracking 
issue couldn't be optimized specifically for the current states compared with MPC, but the vehicle was still under control.

However, when the velocity increased to $11 \mathrm{~m} / \mathrm{s}$, from the Figure 11, it can be seen that the vehicle controlled by MPC was out of control that means the controller couldn't track the expected trajectory anymore. On the contrary, RSC showed a more robust performance though the vehicle still slipped and the vehicle tracked the expected trajectory in the end. Figure 12 demonstrates the simulation results of acceleration. It can be found that compared with RSC, the vehicle controlled by MPC was in critical states for a significantly long time. If the vehicle was in a critical states for a long time, the state information used in its prediction model may be inaccurate as the system was prone to be unstable at this time, which will cause the prediction to fail. For RSC, the REMS of path tracking error is $0.04690 \mathrm{~m}$ in this case. Thus, from the analysis above, it can be concluded that the maximum velocity MPC can carry out was $10 \mathrm{~m} / \mathrm{s}$. Next, the velocity will be increased for the test of RSC. The step applied in this simulation was $1 \mathrm{~m} / \mathrm{s}$.

To simplify, the simulation results of $12 \mathrm{~m} / \mathrm{s}$ and $13 \mathrm{~m} / \mathrm{s}$ were not displayed here. Figures 13 and 14 display the simulation results of RSC when the velocity was $14 \mathrm{~m} / \mathrm{s}$ and $15 \mathrm{~m} / \mathrm{s}$, respectively. Obviously, the vehicle was out of control when the velocity was $15 \mathrm{~m} / \mathrm{s}$. When the expected velocity was $14 \mathrm{~m} / \mathrm{s}$, the vehicle tracked trajectory after experiencing slipping. Besides, it can be found that the period that acceleration exceeded to $0.2 g$ is shorter in Figure 15 compared with Figure 16. Therefore, the vehicle was out of control when the velocity was increased to $15 \mathrm{~m} / \mathrm{s}$.

\subsection{Case 3: Extreme Curve Test}

The goal of this section is to study the performance of MPC and RSC when the road adhesion and road curvature change abruptly. Thus, as shown in Figure 17, a curve road was built in CarSim, the maximum curvature was set as $0.02 \mathrm{~m}^{-1}$, the road adhesion was set as 0.85 if global $\mathrm{X}$ was in the range of $[0,20 \mathrm{~m}]$, otherwise, it was set as 0.2 . Similarly, the initial lateral, angular and velocity errors of AVs were all set as 0 .

When the velocity was set as $9 \mathrm{~m} / \mathrm{s}$, the simulation results are shown in Figures 18 and 19. Both MPC controller and RSC controller tracked the expected trajectory well, where the REMS of path tracking error is $0.025 \mathrm{~m}$ and $0.0412 \mathrm{~m}$, respectively. From Figure 19, it can be found that the maximum acceleration didn't exceed $0.2 g$, so it can be considered that the current conditions were in the stable regions of both controllers.

However, the vehicle controlled by MPC was out of control when the velocity was up to $10 \mathrm{~m} / \mathrm{s}$ on this kind of roads. The results are shown in Figures 20 and 21, and it's obvious that the vehicle controlled by MPC deviated seriously from the road and the controller lost its stability finally. From Figure 21, it can be known that the vehicle controlled by MPC and RSC slipped from $3 \mathrm{~s}$ to $7 \mathrm{~s}$, but vehicle controlled by MPC was out of control in the end. On the other hand, though the vehicle controlled by RSC also experiencing slipping for a long time, it tracked the expected trajectory in the end, where the REMS of path tracking error is $0.8266 \mathrm{~m}$. Therefore, it can be concluded that if the system is in an unstable state for a long time, RSC will show better performance.

With the purpose to test the maximum velocity that RSC can carry out in this case, we set the velocity as $11 \mathrm{~m} / \mathrm{s}, 12 \mathrm{~m} / \mathrm{s}$ and $13 \mathrm{~m} / \mathrm{s}$, respectively. Figures 22,23 and 24 demonstrate the simulation results of three test. In the tests where velocity was set as $11 \mathrm{~m} / \mathrm{s}$ and $12 \mathrm{~m} / \mathrm{s}$, though the RSC controller wasn't out of control, the tracking performance got worse and worse as velocity increased. When the velocity increased to $13 \mathrm{~m} / \mathrm{s}$, the RSC controller couldn't track the expected trajectory anymore and it lost stability quickly. Figures 25, 26 and 27 show the acceleration profiles of three tests.

Overall, in this case, the maximum velocity MPC can carry out is $9 \mathrm{~m} / \mathrm{s}$, while that of RSC is $12 \mathrm{~m} / \mathrm{s}$.

\section{Conclusions}

1. A comparative study of MPC and RSC techniques for trajectory tracking was presented. The tracking model with 3-DOF vehicle dynamics and linear tire model, was deduced.

2. The MPC-based and RSC-based trajectory tracking controller were designed based on the same longitudinal-yaw-lateral motions of a single-track vehicle with a linear tire model, respectively. With the purpose to compare the discrepancy between them, three scenarios were built in CarSim, including verification test, double lane change test and curve test.

3. The simulation results show that MPC controller performed better in term of accuracy and responding time when the driving conditions was well. In the test of double lane change manoeuvre where the road adhesion was set as 0.2 , the maximum velocity MPC can carry out was only $10 \mathrm{~m} / \mathrm{s}$, while that RSC can execute was $14 \mathrm{~m} / \mathrm{s}$. In the curve test with curvature $0.02 \mathrm{~m}^{-1}$ where road adhesion changes abruptly from 0.85 to 0.2 , the maximum velocity MPC can carry out was $9 \mathrm{~m} / \mathrm{s}$ and that for RSC was $12 \mathrm{~m} / \mathrm{s}$.

4. In can be concluded that RSC is robust and can reach a higher velocity when the driving conditions becomes worse, while MPC is prone to be unstable. 


\section{Acknowledgements}

The authors sincerely thanks to Dr. Wenhao Yu of Tsinghua University and Dr. Xin Yang of Chongqing University for their critical discussion and reading during manuscript preparation.

\section{Authors' Contributions}

$X T$ was in charge of the whole trial; KY wrote the manuscript; $Y Q, Y H, H W$ and HP assisted with sampling and laboratory analyses. All authors read and approved the final manuscript.

\section{Authors' Information}

Kai Yang is currently pursuing the Ph.D. degree at College of Mechanical and Vehicle Engineering, Chongaing University, China.

Xiaolin Tang is currently an associate professor at College of Mechanical and Vehicle Engineering, Chongaing University, Chongaing, China. He received the Ph.D. degree in Mechanical Engineering from Shanghai Jiao Tong University, China, in 2015.

Yechen Qin is currently working as an associate professor at Beijing Institute of Technology, China and he is also a visiting scholar at The State Key Laboratory of Mechanical Transmissions, China. He received the B.Sc. and Ph.D. degrees in mechanical engineering from Beijing Institute of Technology, China, in 2010 and 2016, respectively.

Yanjun Huang is currently a research professor at School of Automotive Studies, Tongji University, Shanghai. He received his PhD degree at Department of Mechanical and Mechatronics Engineering at University of Waterloo, Canada in 2016.

Hong Wang is currently an associate professor at School of Vehicle and Mobility Tsinghua University, China. She received the Ph.D. degree from Beijing Institute of Technology, China, in 2015.

Huayan Pu, is currently a professor at State Key Laboratory of Mechanical Transmissions, Chongqing University, China. She received the Ph.D. degree from Huazhong University of Science and Technology, China, in 2011.

\section{Funding}

Supported by Natural Science Foundation of China (Grant Nos. 52072051, 51705044), Chongqing Municipal Natural Science Foundation of China (Grant No. cstc2020jcyj-msxmX0956), State Key Laboratory of Mechanical System and Vibration (Grant No. MSV202016), and State Key Laboratory of Mechanical Transmissions (Grant No. SKLMT-KFKT-201806).

\section{Competing Interests}

The authors declare no competing financial interests.

\section{Author Details}

${ }^{1}$ College of Mechanical and Vehicle Engineering, Chongqing University, Chongqing 400000, China. ${ }^{2}$ School of Mechanical Engineering, Beijing Institute of Technology, Beijing 100080, China. ${ }^{3}$ School of Automotive Studies, Tongji University, Shanghai 201804, China. ${ }^{4}$ School of Vehicle and Mobility, Tsinghua University, Beijing 100080, China.

Received: 14 May 2020 Revised: 3 June 2021 Accepted: 13 July 2021 Published online: 12 August 2021

\section{References}

[1] D González, J Pérez, V Milanés, et al. A review of motion planning techniques for automated vehicles. IEEE Transactions on Intelligent Transportation Systems, 2015, 17(4): 1135-1145.

[2] Y Qin, X Tang, T Jia, et al. Noise and vibration suppression in hybrid electric vehicles: state of the art and challenges. Renewable and Sustainable Energy Reviews, 2020, 124: 109782.

[3] X Tang, S Li, H Wang, et al. Research on energy control strategy based on hierarchical model predictive control in connected environment. Journal of Mechanical Engineering, 2020, 56(14): 119-128. (in Chinese)
[4] T Liu, XTang, H Wang, et al. Adaptive hierarchical energy management design for a plug-in hybrid electric vehicle. IEEE Transactions on Vehicular Technology, 2019, 68(12): 11513-11522.

[5] C Katrakazas, M Quddus, W H Chen, et al. Real-time motion planning methods for autonomous on-road driving: State-of-the-art and future research directions. Transportation Research Part C: Emerging Technologies, 2015, 60: 416-442

[6] XYang, X Tang, KYang, et al. Local motion planning framework for autonomous vehicle considering position uncertainty in highway. 2020 4th CAA International Conference on Vehicular Control and Intelligence (CVCI), Hangzhou, China, December, 2020: 471-474.

[7] R Yang, R Xiong, F C Sun. Experimental platform development and characteristics analysis of external short circuit in lithium-ion batteries. Journal of Electrical Engineering. 2021, 16(1): 103-118. (in Chinese)

[8] B Paden, M Čáp, S Yong, et al. A survey of motion planning and control techniques for self-driving urban vehicles. IEEE Transactions on Intelligent Vehicles, 2016, 1(1): 33-35.

[9] Y Huang, HWang, A Khajepour, et al. A novel local motion planning framework for autonomous vehicles based on resistance network and model predictive control. IEEE Transactions on Vehicular Technology, 2019, 69(1): 55-66.

[10] C Hu, H Jing, R R Wang, et al. Robust $\mathrm{H} \infty$ output-feedback control for path following of autonomous ground vehicles. Mechanical Systems and Signal Processing, 2016, 70: 414-427.

[11] M Samuel, M Hussein, M B Mohamad. A review of some pure-pursuit based path tracking techniques for control of autonomous vehicle. International Journal of Computer Applications, 2016, 135(1): 35-38.

[12] S Xu, H Peng. Design, analysis, and experiments of preview path tracking control for autonomous vehicles. IEEE Transactions on Intelligent Transportation Systems, 2019, 21(1): 48-58.

[13] Y Huang, H Ding, Y Zhang, et al. A motion planning and tracking framework for autonomous vehicles based on artificial potential field elaborated resistance network approach. IEEE Transactions on Industrial Electronics, 2019, 67(2): 1376-1386.

[14] Y Zhang, X Wang, $\mathrm{H}$ Yang, et al. Robust predictive current control of induction motors based on linear extended state observer. Chinese Journal of Electrical Engineering, 2021, 7(1): 94-105.

[15] R Rajamani. Vehicle dynamics and control. Springer Science \& Business Media, 2011.

[16] R Attia, R Orjuela, M Basset. Combined longitudinal and lateral control for automated vehicle guidance. Vehicle System Dynamics, 2014, 52(2): 261-279.

[17] F Borrelli, P Falcone, T Keviczky, et al. MPC-based approach to active steering for autonomous vehicle systems. International Journal of Vehicle Autonomous Systems, 2005, 3(2): 265-291.

[18] P Falcone, F Borrelli, J Asgari, et al. Predictive active steering control for autonomous vehicle systems. IEEE Transactions on Control Systems Technology, 2007, 15(3): 566-580

[19] J Ji, A Khajepour, W Melek, et al. Path planning and tracking for vehicle collision avoidance based on model predictive control with multi-constraints. IEEE Transactions on Vehicular Technology, 2016, 66(2): 952-964.

[20] L Chen, H Nian, Y X. Improved model predictive direct power control of grid side converter in weak grid using Kalman filter and DSOGI. Chinese Journal of Electrical Engineering, 2019, 5(4): 22-32.

[21] J Guo, Y Luo, K Li. Robust gain-scheduling automatic steering control of unmanned ground vehicles under velocity-varying motion. Vehicle System Dynamics, 2019, 57(4): 595-616.

[22] L Shen, H Xiao. Delay-dependent robust stability analysis of power systems with PID controller. Chinese Journal of Electrical Engineering, 2019 5(2): 79-86.

[23] D Q Mayne. Model predictive control: Recent developments and future promise. Automatica, 2014, 50(12): 2967-2986.

[24] F Yakub, Y Mori. Comparative study of autonomous path-following vehicle control via model predictive control and linear quadratic control. Proceedings of the Institution of Mechanical Engineers, Part D: Journal of Automobile Engineering, 2015, 229(12): 1695-1714.

[25] D Q Mayne, J B Rawlings, C V Rao, et al. Constrained model predictive control: Stability and optimality. Automatica, 2000, 36(6): 789-814.

[26] X L Tang, T Jia, X S Hu, et al. Naturalistic data-driven predictive energy management for plug-in hybrid electric vehicles. IEEE Transactions on Transportation Electrification, 2021, 7(2): 497-508. 
[27] N Wada, H Tomosugi, M Saeki. Model predictive tracking control for a linear system under time-varying input constraints. International Journal of Robust and Nonlinear Control, 2013, 23(9): 945-964.

[28] C Hu, L Zhao, L Cao, et al. Steering control based on model predictive control for obstacle avoidance of unmanned ground vehicle. Measurement and Control, 2020, 53(3-4): 501-518.

[29] R Wang, $\mathrm{H}$ Jing, $\mathrm{CHu}$, et al. Robust $\mathrm{H} \infty$ path following control for autonomous ground vehicles with delay and data dropout. IEEE Transactions on Intelligent Transportation Systems, 2016, 17(7): 2042-2050.

[30] H Zhang, X Zhang, JWang. Robust gain-scheduling energy-to-peak control of vehicle lateral dynamics stabilisation. Vehicle System Dynamics, 2014, 52(3): 309-340.

[31] H Du, N Zhang, F Naghdy. Velocity-dependent robust control for improving vehicle lateral dynamics. Transportation Research Part C: Emerging Technologies, 2011, 19(3): 454-468
[32] X Huang, $\mathrm{H}$ Zhang, G Zhang, et al. Robust weighted gain-scheduling $\mathrm{H} \infty$ vehicle lateral motion control with considerations of steering system backlash-type hysteresis. IEEE Transactions on Control Systems Technology, 2014, 22(5): 1740-1753.

[33] Y Ren. Research on active tracking and collision avoidance control of intelligent electric vehicles. Chongqing: Chongqing University, 2018. (in Chinese)

[34] S Boyd, E L Ghaoui, E Feron, et al. Linear matrix inequalities in system and control theory. Society for Industrial and Applied Mathematics, Philadelphia, USA, 1994.

\section{Submit your manuscript to a SpringerOpen ${ }^{\circ}$ journal and benefit from:}

- Convenient online submission

- Rigorous peer review

- Open access: articles freely available online

- High visibility within the field

- Retaining the copyright to your article

Submit your next manuscript at $\boldsymbol{\nabla}$ springeropen.com 